\title{
Microbubble Distillation Studies of a Binary Mixture
}

\author{
A. Al-Yaqoobi, W. B. Zimmerman \\ Department of Chemical and Biological Engineering, Faculty of Engineering, University of Sheffield
}

\begin{abstract}
The characteristics of microbubble distillation of binary system of ethanol and water have been investigated. The study describes the use of a fluidic oscillator used to generate microbubbles. The effects of air temperature and liquid level on separation performance have been studied. The results demonstrate that ethanol concentration in the distilled phase decreases with increasing liquid level. Increasing air temperature enhances the separation efficiency of the two components. It is shown that the ethanol fraction in distilled phase and the evaporation ratio increases by increasing the air temperature. Furthermore, the concentration of ethanol in the residual liquid shows a corresponding decrease. The vapour-liquid composition of microbubble distillation can exceed the isothermal equilibrium vapour-liquid composition by controlling the liquid level and the air temperature within the process.
\end{abstract}

Keywords Binary Mixture; Direct Contact Evaporation; Fluidic Oscillator; Microbubble Distillation; Microbubbles

\section{INTRODUCTION}

Phase transition can be defined explicitly by temperature and pressure in binary liquid solutions. Energy is added to raise the liquid temperature of the mixture to boiling point to achieve high vapour pressure of the volatile component, which is a direct function of the vapour temperature. Consequently, the vapour phase will be richer in the more volatile component than the original liquid mixture. Fractional distillation is used to increase the purity of the vapour phase. This is enhanced by increasing the contact surface area between the hot vapour and the cold liquid.

Recently, a computational model has been developed to study the evaporation dynamic of microbubbles in air-water systems. This hypothesis is achieved by taking into consideration the internal gas dynamics of the bubble [1]. The study questions the concept of heating the gas phase rather than the liquid phase to achieve the maximum equilibrium vapour pressure as in traditional distillation. The model also explores the effect of microbubbles on the mechanisms of sensible heat transfer and evaporation. Since microbubbles have high surface area to volume ratio, both sensible heat transfer and evaporation rates are affected by the high gas-liquid interfacial contact area. The model shows that maximum evaporation can be achieved in a short bubble resistance time. Further contact between the bubble and surrounding liquid leads to sensible heat transfer and cooling of the bubble accompanied by condensation of vapour back into the liquid phase, effectively achieving a dynamic equilibrium.

Via judicious control of reaction conditions it is possible to achieve separation of the components within the mixture using this microbubble distillation technology. This could lead to the separation of the components within the mixture on a much more efficient basis.

The current study is focused on the separation of ethanol and water, where the dynamics of the gas microbubbles are explored as the main area of study. The fluidic oscillator [2] is used in all experiments to generate microbubbles. The aim of this work is to study the effect of liquid level and air temperature on microbubble distillation performance.

\section{EXPERIMENTAL APPARATUS AND MATERIAL}

The experiments were performed using the batch system shown in Fig. 1. The air stream was heated and fed via the fluidic oscillator into the tank through a microporous diffuser. The flow rate of air was controlled by bleed valves which regulate the overall gas flow. The actual flow rate was measured via a flow meter at the tank gas exit. The outlet stream was connected to a glass condenser which was cooled by ice/salt coolant to maintain the condenser between -19 to $-20^{\circ} \mathrm{C}$. Before the liquid was poured into the tank, the system was heated for approximately 60 minutes. Following the completion of each experiment the residual liquid was collected and final volume was measured to calculate the evaporation ratio (EV \%) as in (1). All the samples were analysed by Bruker's ALPHA FTIR-ATR spectrometer.

$$
\mathrm{EV} \%=\frac{\text { Intial volume }- \text { final volume }}{\text { Intial volume }}
$$

$\mathrm{Xf}$ is the concentration of residual liquid, $\mathrm{Xc}_{\mathrm{c}}$ the concentration of distilled fraction, and EV\% the evaporation ratio.

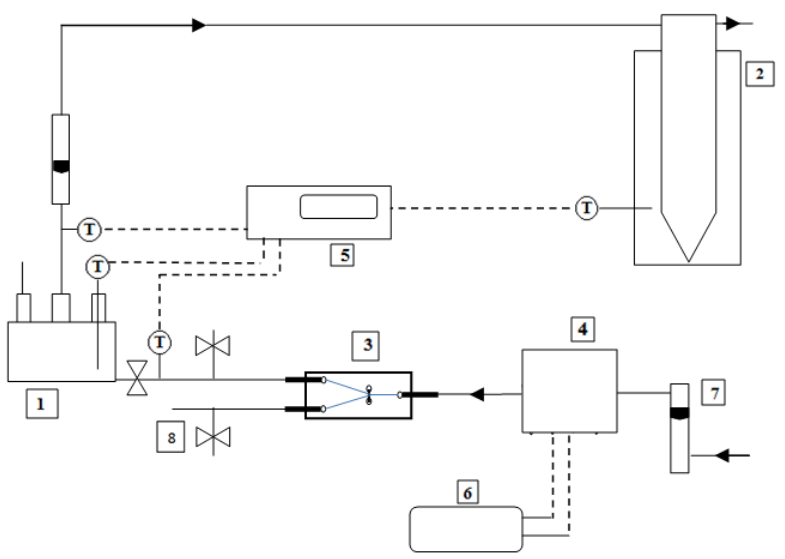

Figure 1. Schematic diagram of the experimental set up. 1: Separation Tank, 2: Condenser, 3: Fluidic oscillator, 4: Air heater, 5: Microprocessor, thermometer, 6: Temperature controller, 7: Rotameter, 8: Bleeding valves. 


\section{RESULT AND DISCUSSION}

\subsection{EFFECT OF LIQUID LEVEL}

The effect of liquid level was studied with liquid concentration of $40 \%$ with air temperature of $120^{\circ} \mathrm{C}$. Fig. 2 shows that Xc decreased from about $78 \%$ at liquid levels of $2 \mathrm{~mm}$ to around $76 \%$ at $40 \mathrm{~mm}$. After a short period of bubble detachment from the diffuser, evaporation dominates and ethanol has reached the maximum concentration which is proportional to bubble interface temperature. Thereafter, ethanol is depleted from the layer surrounding the gas-liquid interface before the bubble reaches thermodynamic equilibrium. The ethanol concentration in the vapour phase reduces as the bubble rises in the liquid due to the preferential enrichment of the vapour-liquid interface by water molecules. The last fraction accumulated in the condenser at liquid level of $2 \mathrm{~mm}$ was $69 \%$ and around 75.8 for $40 \mathrm{~mm}$. That gives a partition coefficient $(K=X c / X f)$ range from 4.3 at $2 \mathrm{~mm}$ to 1.96 at 40 $\mathrm{mm}$, indicating that a high level of non-equilibrium separation is achievable.

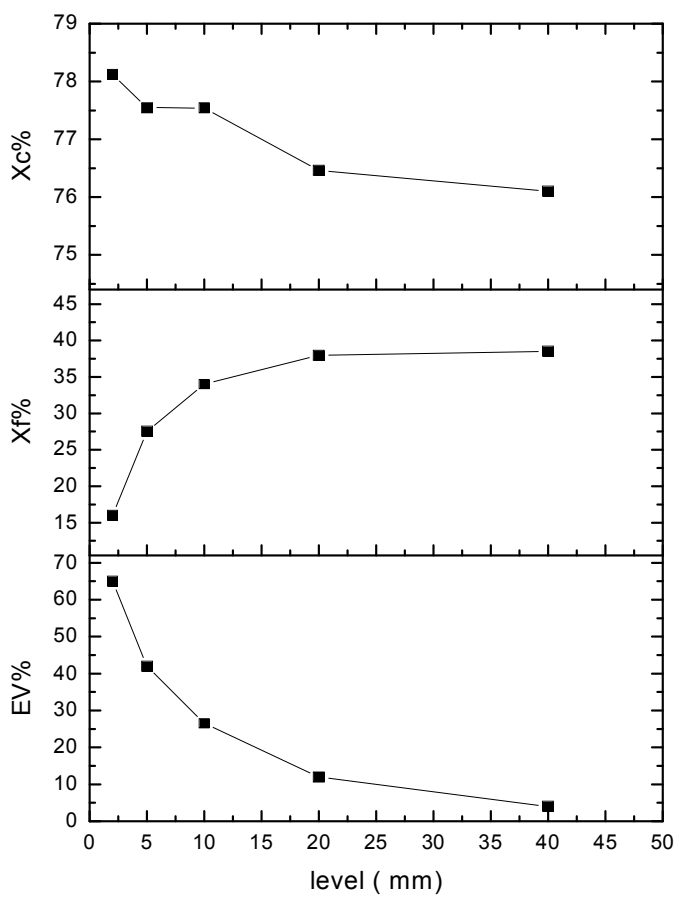

Figure 8. The effect of liquid level. The $\left(X_{c}\right)$ values shown are for the first samples accumulated in the condenser. The $(\mathrm{Xf})$ values are the concentration of residual liquid after the completion of the experiment.

\subsection{Effect OF Air Temperature}

The results plotted in Fig. 3 show the effect of air temperature with initial liquid concentration of $40 \mathrm{wt} \%$ and liquid level of $5 \mathrm{~mm}$. As the air temperature increased from 50 to $120^{\circ} \mathrm{C}$, the $\mathrm{Xc}$ value increased from $75.6 \%$ to around $77.5 \%$. Concurrently, the residual liquid concentration (Xf) shows a decrease from $30.4 \%$ to around $27.5 \%$. In addition, the evaporation ratio $\mathrm{EV} \%$ increased from $29 \%$ to $45 \%$. The concentration of ethanol in the vapour phase is propositional to its saturated vapour pressure at the bubble interface; the latter is a function of the bubble temperature. Thus, the amount of ethanol transferred from liquid to the vapour phase increases as the air temperature is elevated. The liquid temperature slowly increases with a corresponding increase in the air temperature during the experimental time.

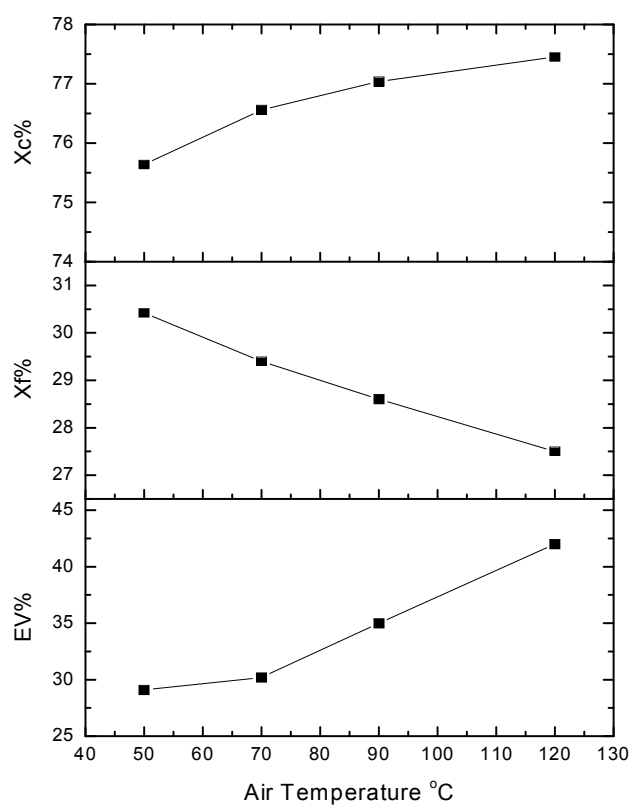

Figure 9. The effect of air temperature. The (Xc) values shown are for the first samples accumulated in the condenser. The (Xf) values are the concentration of residual liquid after the completion of the experiment.

\section{CONCLUSIONS}

From the results achieved to date the use of microbubble distillation is a promising future technology for the separation of binary mixtures as described. Using microbubble distillation, maximum evaporation of ethanol can be achieved at low liquid level. It was shown that the partition coefficient decreased from 4.3 to 1.9 when the liquid level increases from $2 \mathrm{~mm}$ to $40 \mathrm{~mm}$ at air temperature of $120^{\circ} \mathrm{C}$. It was also shown that by increasing the air temperature, the evaporation ratio and percentage of ethanol in distilled phase Xc was increased.

\section{ACKNOWLEDGEMENTS}

The authors would like to thank the Iraqi Ministry of Higher Education and scientific research for a doctoral scholarship; Dr. David Hogg for helpful discussion and chemical analysis.

\section{REFERENCES}

1. Zimmerman WB, Al-Mashhadani MKH, Bandulasena $\mathrm{HCH}$. Evaporation dynamics of microbubbles. Chemical Engineering Science. 2013;101(0):865-77.

2. Zimmerman $\mathrm{WB}$, Tesar $\mathrm{V}$, Butler $\mathrm{S}$, Bandulasena $\mathrm{HCH}$. Microbubble generation. Recent Patents on Engineering. 2008;2(1):1-8. 